\title{
A Trajetória de "Arquivos de Biologia e Tecnologia", Publicação que Marcou Época na História da Ciência Brasileira
}

\author{
Metry Bacila \\ Ex-Chefe do Serviço de Bioquímica e da Divisão de Patologia Experimental do Instituto de Biologia e Pesquisas \\ Tecnológicas
}

\section{REPOSITÓRIO HISTÓRICO}

Ao completar cinquienta e cinco anos de ininterrupta publicação, Arquivos de Biologia e Tecnologia, hoje no formato de prestigiosa publicação internacional, como Brazilian Journal of Biology and Technology, marcou e, certamente, vem marcando extraordinário exemplo de vitalidade científica, raramente encontrado entre publicações dessa natureza. Alteia-se, assim, Arquivos, a prestigiosas revistas científicas do País e do exterior, que vem registrando, de modo ininterrupto, o que de mais básico e fundamental a pesquisa científica vem conquistando em benefício do homem.

Arquivos de Biologia e Tecnologia traz, escondida entre suas vetustas páginas, a própria história do IBPT, o nosso Instituto de Biologia e Pesquisas Tecnológicas de tantas lutas, de tantas glórias e de tantas rememorações, hoje transformado em Instituto de Tecnologia do Paraná, TECPAR, mantendo, contudo, o espírito, os propósitos, as metas e os objetivos que nortearam a criação e a implantação do IBPT de Marcos Augusto Enrietti, lá pelos idos de 1940-1942.

Deve-se ao Prof. Marcos Augusto Enrietti, na realidade o Dr. Marcos de todos os que tiveram o privilégio de conviver com ele nas afanosas lides científicas do IBPT, a iniciativa, o descortino e o pioneirismo de criar uma instituição científica dedicada ao desenvolvimento do Estado do Paraná Em 1940, com o apoio do Governador Manoel Ribas, Marcos Augusto Enrietti funda o Laboratório de Análises da Secretaria de Viação, Obras Públicas e Agricultura, passando, em 1941, a Instituto de Biologia Agrícola e Animal e, em 1942, considerando que suas finalidades transcendiam dos campos da agricultura e da veterinária, a Instituto de Biologia e Pesquisas Tecnológicas, o nosso IBPT, com uma atuação mais abrangente e mais ampla, já que tinha, como objetivos, fomentar, propugnar e concorrer para o desenvolvimento da pecuária, da agricultura e da indústria do Estado.

Graças à extraordinária visão de Marcos Augusto Enrietti, o IBPT alcançou sui generis organização em todo o Brasil, dotando-o de infra-estrutura técnica, científica e administrativa capaz de atender à pesquisa científica e tecnológica em todos os seu aspectos, com as Divisões de Biologia Vegetal, Biologia Animal, Patologia Experimental, Química e Tecnologia, Geologia e Mineralogia, Solos, Metrologia, Experimental de Combustíveis, além da Divisão Administrativa, esta englobando toda a parte operativa do IBPT, inclusive a Contabilidade, o Almoxarifado, o Serviço de Transportes.

Ao tempo da criação do IBPT, a Universidade do Paraná, a mais antiga Universidade brasileira, 
então desmembrada em suas diferentes Faculdades, contava já com 28 anos de existência, fundada que fora por Victor Ferreira do Amaral e Silva e seus ilustres companheiros dessa heróica empreitada, em 1912.

Por outro lado, o Governo do Estado cria, em 1941, o Instituto Técnico de Agronomia, Veterinária e Química, reunindo os seus respectivos cursos, em estabelecimento único, ocupando a antiga Escola Rural Carlos Cavalcanti, no Juvevê, ao lado dos próprios do IBPT. Certamente, contava a Universidade do Paraná com expressivos nomes de mestres em suas diversas áreas de atuação, como foram, entre outros, os Professores Archimedes de Oliveira e Cruz, na área da Farmacologia, Antenor Pamphilo dos Santos, em Fisiologia, Maria Falce de Macedo, em Química Fisiológica, Arthur Otto Schwab, em Biofísica, indubitavelmente, o maior talento científico jamais surgido entre nós e cuja biografia ainda está por ser escrita. Com o que não contava, porém, o ensino superior da época era com uma infra-estrutura de pesquisa científica do nível e do porte da que o Prof. Marcos Augusto Enrietti construiu, organizou e tornou uma realidade altamente significativa na forma do Instituto de Biologia e Pesquisas Tecnológicas.

Apesar de constituir-se em órgão do Governo do Estado, exerceu o IBPT, extraordinária influência na área da pesquisa científica entre nós e, certamente, do ensino superior também, já que a quase totalidade de seus pesquisadores científicos era constituída de membros do corpo docente de diferentes unidades de ensino superior da Universidade do Paraná bem como do Instituto Técnico de Agronomia, Veterinária e Química cursos estes posteriormente integrados à Universidade, tendo sido, assim fator fundamental para a Restauração da Universidade do Paraná, em 1946, quando Ministro da Educação o Prof. Ernesto Souza Campos, e para sua posterior federalização. Contava o IBPT, com extraordinária plêiade de pesquisadores científicos e de técnicos e funcionários da mais alta categoria e elevado padrão: Heitor Segundo Guilherme Medina, Reinhard Maack, Lycio Grein de Castro Vellozo, Milton Geovanoni, Alsedo Leprevost, Ludwig Johann Weber, Reinaldo Krauze Spitzner, Nilton Emilio Bührer, Carlos Bodziak Jr. Dirceu Correia, Oscar Krebs Palmquist, Gastão Victor
Langmann Kubiak, Teturo Yamada, David da Silva Carneiro Jr., Mário José Nowacki, Angelo Molfi, Mario De Lavigne, Pedro Costa Muniz, Oswaldo Augusto Wendler, Oswaldo Fontoura, Milton Miró Vernalha, Oswaldo Ceccon, Christian Bomskov, Guenter Tessman, Wladimir Cavallar Kavaleridze, Julio C. Felix, Fridolin Schlögel, Orisel Curial, John Augustus Brown, Ruy Santos, Astolpho Macedo de Souza Filho, Álvaro Pereira Jorge, Dinor Olegário Voss, Dilermando Britto, José Paim de Andrade, Ivan Austregésilo Maida, Cecília Egg, Renata Berner, Milla Aguillar, Percy Spitzner, João José Bigarella, Olavo Romanus, Ruy Santos, Aroldo Frenzel, José Milton Andriguetto, Léo da Rocha Lima, Yasuyoshi Hayashi, Salvador Fernandes Neto, Darci Biagini, Manoel Motzko, os dois últimos competentes auxiliares técnicos, e tantos outros, bem como funcionários da mais alta categoria, como o casal D. Yone e Ubaldino, o exemplar e dedicado Theodoro, o nosso sempre lembrado Alcebíades, e aquele extraordinário artista e desenhista técnico do IBPT, Augusto Comte, criador dos emblemas do Instituto.

Tópico especial deve ser dedicado, nestas rememorações, à "vertente bioquímica" do IBPT, tendo em conta a grande ênfase que foi dada à pesquisa bioquímica, inicialmente na Divisão de Patologia Experimental, da qual fui honrado com a nomeação para sua Chefia por Marcos Augusto Enrietti, e depois na Divisão de Bioquímica, atividade essa que acabou por tornar-se uma das mais prestigiosas do País. Integravam o grupo de seus pesquisadores na área da Bioquímica, por nós implantada no IBPT, Dinor Olegário Voss, Luiz Alberto Silva Veiga, Alceu Schwab, José Hazencleve Duarte, Déa Amaral, Glaci Zancan, Emilia Slobodian Mota, Doracy Passos, Clotilde de Lourdes Branco Germiniani, Teresinha Cardoso, Annibal de Paiva Campello, Sieg Odebrecht, Jair Campello, Alexandre Dmytraczenko, Norma Stenzel, nossa competente Secretária, com a colaboração inestimável de Heitor Medina. Com um ativíssimo programa de pesquisas e, mais ainda, com a criação do Curso de Fisiologia de Microorganismos, de grande notoriedade nacional e internacional, com atividades de pós-graduação nessa área e, ainda, com a criação do Instituto de Bioquímica da Universidade Federal do Paraná, uma iniciativa do nosso grupo de pesquisadores do 
IBPT, e a implantação do primeiro Mestrado da UFPR, a "vertente bioquímica" do IBPT foi de tal forma significativa que se traduziu em considerável número de contribuições científicas publicadas em Arquivos de Biologia e Tecnologia, seu principal órgão de registro dos resultados das pesquisas conduzidas no IBPT, bem como de trabalhos oriundos das atividades desenvolvidas pelo Curso de Fisiologia de Microorganismos.

Fato semelhante ocorreu com as atividades levadas a efeito pelos pesquisadores de todas as áreas de perquirição científica e tecnológica desenvolvidas nos diversas Divisões que compunham o IBPT, registradas que foram em Arquivos de Biologia e Tecnologia

No que concerne à área administrativa, muito deve o sucesso do IBPT a uma plêiade de notáveis funcionários como Alexandre Zainko, aliás, autor do primeiro Boletim Técnico do IBPT, Chefe da Seção Administrativa, depois substituído por Dionízio Grabowski, Hamilcar Ceccato, Odimar Bührer, Neuzarth Machado e tantos outros.

Menção especialíssima cabe aqui, quando se rememora a saga histórica de Arquivos de Biologia e Tecnologia, à nossa extraordinária bibliotecária de então, Carlota Ilnicki, a cujo zelo e carinho, muito deve a regularidade na sua publicação e distribuição. Personalidade marcante não só por sua atuação direta nos cuidados da Biblioteca ao seu encargo mas também dotada de invejável grau de cultura e de conhecimentos científicos, Carlota Ilniski mereceu de todos os que a conheceram em seu importante mister, respeito e consideração.

\section{O IBPT, A SOCIEDADE BRASILEIRA PARA O PROGRESSO DA CIÊNCIA (SBPC) E A FUNDAÇÃO ROCKEFELLER}

Tendo em mãos ponderável acervo científico Arquivos de Biologia e Tecnologia contava já com quatro volumes publicados - vol. I (1946) ao vol. IV (1949), e já com uma belíssima história científica a relatar, levando na bagagem os extraordinários trabalhos de Heitor Medina, sobre leishmanioses e, mais particularmente, sobre a Leishmania enrietti, os belíssimos trabalhos de Reihnard Maack sobre geologia do Paraná e de Santa Catarina, e os de João José Bigarella sobre a planície litorânea do Estado do Paraná, os estudos sobre os solos dos Campos Gerais no Estado do
Paraná de Carlos Bodziak Jr e Reinhard Maack, as contribuições sobre química analítica inorgânica e orgânica de Reinaldo Krauze Spitzner e de Nilton Emílio Bührer, o fundamental trabalho de Alsedo Leprevost cuidando da análise de calcáreos paranaenses, bem como os trabalhos fundamentais de Milton Giovanoni e Gastão Victor Langmann Kubiak, cuidando da fauna parasitológica paranaense e o de Lycio Grein de Castro Vellozo, Mario José Nowacki e Milton Miró Vernalha, dando conta de levantamento fitossanitário do Estado do Paraná, uma notável contribuição de Oscar K. Palmquist sobre brucelose no Estado do Paran;a, um trabalho de bioquímica enzimológica que marcou o início da "vertente bioquímica" na produção científica do IBPT, de Metry Bacila, sobre galactoquinase em tecidos animais e, last but not least, um belíssimo trabalho do Mestre de todos nós, o Prof. Arthur Otto Schwab, sobre limitações nas medidas físico-químicas, - armado de todo esse potencial científico e de muitos outros mais que compunham os já publicados volumes de Arquivos de Biologia e Tecnologia, o Doutor Marcos comparece à I Reunião da Sociedade Brasileira para o Progresso da Ciência, a nossa SBPC, realizada em Campinas, no Estado de São Paulo. Tive a felicidade de acompanhá-lo nessa histórica missão científica, à qual compareceram os mais representativos cientistas brasileiros, bem como de outros países da América Latina.

Presente à Reunião da SBPC, em Campinas, estava também o Dr. Harry Milton Miller, Jr., ilustre membro da Fundação Rockefeller, com quem o Dr. Marcos manteve cordialíssimo encontro que marcou o início de uma época das mais brilhantes da pesquisa científica no IBPT. Acompanhando o Dr. Marcos em todos esses momentos, tive a oportunidade de testemunhar o apreço e o entusiasmo que geraram as notícias e as informações relativas ao IBPT, sua trajetória como modelar instituição de pesquisa biológica e tecnológica, a sua elogiável infra-estrutura científica, os seus objetivos, bem como a sua produção científica consubstanciada nos já publicados volumes de Arquivos de Biologia e Tecnologia.

As relações do IBPT com a Fundação Rockefeller tiveram aí o seu início, com importantes reflexos para a Universidade do Paraná. Convidado pelo Dr. Marcos, já no ano seguinte, o Dr. Miller vem a 
Curitiba, durante a viagem que encetaria pelo Brasil, permanecendo vários dias em nossa cidade. A visita ao IBPT impressionou-o muito, já que encontrou uma instituição extremamente ativa, dinâmica, com moderna infra-estrutura e contando com um corpo de pesquisadores da mais alta categoria. Com isso, inicia-se uma importante era de "Rockefeller Foundation Fellowships" para o IBPT. Tendo sido agraciado com o primeiro "fellowship", foi-me dada a oportunidade de freqüentar e realizar pesquisas em laboratórios da mais alta qualificação científica nos Estados Unidos, tendo o IBPT recebido, no meu retorno, substancial auxílio financeiro da própria Fundação Rockefeller para adquirir equipamentos científicos modernos destinados às pesquisas bioquímicas levadas a efeito em nossos laboratórios da Divisão de Patologia Experimental.

A partir de então, vários de nossos colegas e colaboradores da Divisão de Patologia Experimental - Heitor Medina, Annibal de Paiva Campello, Luiz Alberto Silva Veiga, Déa Amaral, Dinor Olegário Voss, Glaci Zancan - agraciados, também, com "Rockefeller Foundation Fellowships" deram expressão extraordinária às pesquisas na área da Bioquímica, enriquecendo todo o acervo registrado por Arquivos de Biologia e Tecnologia, hoje um extraordinário repositório de uma história científica de grande significado, inclusive das pesquisas encetadas em áreas como a da bioquímica de planorbídeos, da bioquímica de fungos patogênicos e da fisiologia de microorganismos, bem como de estudos sobre o metabolismo de protozoários do gênero Leishmania, esta última desenvolvida em razão da descoberta por Heitor Medina da Leishmania enrietti.

A Reunião da SBPC de Campinas teve outra importante consequiência: a escolha de Curitiba e, particularmente do Instituto de Biologia e Pesquisas Tecnológicas, para sede da II Reunião Anual da Sociedade Brasileira para o Progresso da Ciência, um notável evento organizado por Marcos Augusto Enrietti e sua equipe de colaboradores do IBPT e que foi coroada de extraordinário êxito com a presença de personalidades como Henrique da Rocha Lima, Adolpho Martins Penha, Clemente Pereira, Maurício da Rocha e Silva, Gilberto Guimarães Villela, André Dreyfus, Paulo Sawaya, Francisco Maffei, Mario Autuori, Haity
Moussatché, Gastão Rosenfeld, Crodowaldo Pavan, José Leal Prado, Oswaldo Frota-Pessoa, C.R. Krug, H. Rheinbolt, F. Rawitscher, Oscar Dondera, de Montevidéo, entre tantos outros eminentes cientistas, acusando comparecimento de 90 por cento dos 258 membros da SBPC,

Pontificaram nessa Reunião vários dos cientistas do IBPT, entre os quais Reinhard Maack, o Prof. Arthur Otto Schwab, Heitor Medina, Ludwig Johan Weber, Alsedo Leprevost, Milton Miró Vernalha, Milton Giovanoni, Lycio Grein de Casto Vellozo, entre outros, tendo havido também uma sessão especial "Sobre alguns dos nossos Institutos de Pesquisa - IPT de São Paulo, o IBPT do Paraná e o Instituto Biológico de São Paulo" durante a qual pronunciaram conferências os doutores Francisco H. Maffei, Marcos Augusto Enrietti e Adolpho Martins Penha. Exposições permanentes foram realizadas durante a Reunião, no IBPT, sobre genética, biologia da saúva, gomose de Phytophtora dos Citrus, e sobre o aproveitamento do xisto pirobetuminoso.

\section{DO BOLETIM TÉCNICO PARA ARQUIVOS DE BIOLOGIA E TECNOLOGIA.}

Defendendo com muito entusiasmo o ponto de vista de que para cumprir suas reais finalidades, uma entidade científica deve manter elo de ligação com a comunidade, através de publicações especializadas, cria o Dr. Marcos, já em 1941, ainda sob a égide do Instituto de Biologia Agrícola e Animal, uma importante publicação, o Boletim Técnico. Coube a Alexandre Zainko, Chefe da Administração, a elaboração do primeiro Boletim Técnico. justamente sobre o tema central das atividades do IBAA - A ciência a serviço da agropecuária no Governo Manoel Ribas. Em 1942 é fundado o Instituto de Biologia e Pesquisas Tecnológicas, que edita mais 13 números do Boletim, até 1946, quando, considerando que a produção científica do Instituto comportava já uma revista científica destinada a registrar os resultados obtidos pela intensa atividade de pesquisa que tinha curso nas diversas Divisões de que se constituía o IBPT, funda, o Dr. Marcos, os Arquivos de Biologia e Tecnologia, sem interromper o Boletim Técnico, atualmente já com 67 números publicados. 
Passados 55 anos de seu volume número 1, Arquivos de Biologia e Tecnologia assumiu a forma de prestigiosa revista internacional, Brazilian Archives of Biology and Technology, hoje contemplada com o apoio institucional da Fundação Araucária e do $\mathrm{CNPq}$ e indexada nas mais consideradas fontes de informações internacionais: Biological Abstract and Zoological Records, Biology and Environmental Sciences, Chemical Abstracts, Current Contents/Agriculture, SCI-Science Citation Index, Periodica (Indice de Revistas Latino-Americanas em Ciencias), Ulrich's International Periodicals Directory, Article Clearinghouse.

É muito grato para todos nós que acompanhamos a trajetória de nosso Arquivos de Biologia e Tecnologia, ao longo de sua evolução até a forma atual de Brazilian Archives of Biology and Technology, que entre as 5.722 revistas de todo o mundo indexadas no Science Citation Index - ICS, apenas 16 são brasileiras, constando entre elas o nosso Brazilian Archives of Biology and Technology. Por outro lado, o Institute for Scientific Information - ISI, entidade norteamericana de critérios muito rígidos para a indexação de revistas científicas, listou as publicações mais relevantes para o progresso do conhecimento científico abrangendo cerca de 7000 publicações de todo o mundo, dentre as quais, 21 brasileiras, figurando entre elas, com muito destaque, o nosso Brazilian Archives of Biology and Technology.

\section{A SAGA DE UMA REVISTA CIENTÍFICA}

Arquivos de Biologia e Tecnologia teve o seu primeiro volume (vol. I) publicado em 1946. Não havia um Conselho Editorial nos moldes do que ocorre hoje. Na contracapa a informação seguinte:

Os ABT são publicados anualmente sob a orientação de uma Comissão, respeitadas as opiniões emitidas pelos autores, e adquirida por permuta ou compra. Diretor: Dr. Marcos Augusto Enrietti. Correspondência: Instituto de Biologia e Pesquisas Tecnológicas, Caixa Postal, 357, Curitiba, Paraná, Brasil. Endereço telegráfico: Agribiologia.

The ABT (Biological and Technological Archives) were published annually, under orientation of a
Committee, respecting the oppinions emitted by author's and adquired by exchange or purchase. Director: Dr. Marcos Augusto Enrietti. Mail address: Instituto de Biologia e Pesquisas Tecnológicas, Caixa Postal, 357, Curitiba, Paraná, Brasil. Telegraphic address: Agribiologia.

Essa informação foi repetida até o vol. XI, publicado em 1956.

Ocorre, então, um lapso de 10 anos. Em 1966, Arquivos de Biologia e Tecnologia reaparece com o mesmo formato, a mesma capa, com os mesmos dizeres na contracapa, porém, com uma comovente alteração, aparecendo o Dr. Rolando Sallin Zappa Mansur como Diretor, em substituição a Marcos Augusto Enrietti. Já no volume XIII, correspondente a 1967/1970, ainda com o mesmo formato, era Diretor do IBPT o Dr. Alsedo Leprevost.

Revista IBPT. O que teria ocorrido no período de 1956 a 1966, justamente um lapso de 10 anos na publicação de Arquivos de Biologia e Tecnologia? É que em 1952, o Dr. Marcos Augusto Enrietti funda uma segunda publicação no IBPT, destinada a divulgar para o grande público, notícias e informações técnicas de interesse geral Com isso, aparece uma publicação na forma de revista: IBPT/ Ciência e tecnologia a serviço da produção.

Assuntos como Rotação de culturas, O branqueamento da cera de abelha, A concha de ostra na avicultura, A produção de leite higiênico, A calda bordaleza, Doenças que inutilizam o lavrador, e muitos outros, entre os quais, um sobre Universidades e Institutos Universitários, um tema de grande interesse na época, de sua publicação (1959), integraram a série de artigos aparecidos em "IBPT". Com a denominação de "Revista do IBPT", é ela publicada de 1952 a 1971, quando, naquele que seria o seu número XVII, o Prof. Dinor Olegário Voss, então Diretor do IBPT, resgata a memória de Arquivos de Biologia e Tecnologia, organiza um Conselho Editorial de muito mérito, tendo como Editor Secretário, Ennio Marques Ferreira e como Conselheiros, um importante grupo de pesquisadores científicos do IBPT, da Universidade Federal do Paraná e de entidades científicas e universitárias do País e publica o $\mathrm{n}^{\mathrm{o}} 1$ do vol. XIV (1971) de Arquivos de Biologia e Tecnologia, mantendo, porém, ainda a sigla IBPT, agora encimada por "Arquivos de Biologia e Tecnologia “. 
Assim, no Instituto de Biologia e Pesquisas Tecnológicas, a publicação de Arquivos de Biologia e Tecnologia conviveu de 1952 (vol. VII) até o vol. XI (1956) com a publicação de divulgação técnica - IBPT ou Revista do IBPT.

$\mathrm{O}$ vol. XII de Arquivos de Biologia e Tecnologia foi publicado, ainda, com o mesmo formato tradicional, em 1966, déz anos depois, portanto, do vol. XI.

Nesse interregno o Instituto de Biologia e Pesquisas Tecnológicas manteve a sua atividade divulgadora do conhecimento tecnológico, inclusive na área da biotecnologia, com as publicações IBPT e Revista $I B P T$, até que na sequiência dos números publicados em 1971, reaparece Arquivos de Biologia e Tecnologia, registrado como vol. XIV, ainda mencionado na sua apresentação, como Arquivos de Biologia e Tecnologia/IBPT

Entretanto, já no número seguinte, o Prof. Dinor Olegário Voss resgata, em definitivo, a publicação de Arquivos de Biologia e Tecnologia. Tendo como Editor o Prof. Luiz Alberto Silva Veiga e um Conselho Editorial formado por pesquisadores ilustres do próprio IBPT e de outras entidades científicas do País, o Instituto publica o vol. XV de Arquivos de Biologia e Tecnologia (1972), agora em formato A4, com duas colunas e a capa verde e branca, com uma tarja vertical preta mantendo esse formato até o vol. 39 (1996).

Nesse período - 1972 a 1996 - um detalhe significativo para a história da grande instituição. Quando da publicação do vol.. XV (1972), a capa de Arquivos de Biologia e Tecnologia mantinha, na sarja preta, a sigla IBPT gravada em letras brancas, carcterística que perdurou até o vol. XXI (1978), quando foi substituído pela sigla TECPAR. A grande mudança ocorreu quando da publicação do vol. 40, agora com roupagem azul, cujos números 1,2 e 3 mantinham ainda o título original de Arquivos de Biologia e Tecnologia, transformado que foi, em seu número 4 , em Brazilian Archives of Biology and Technology e cujo Editor é o Prof. Carlos Ricardo Soccol que vem desenvolvendo um trabalho altamente meritório na editoração desse nosso tradicionalíssimo veículo de informação científica. Até o volume XI (1956), Arquivos de Biologia e Tecnologia não contava com um Conselho Editorial. Como já foi mencionado, constava na contracapa de cada volume, que a publicação era da responsabilidade de uma Comissão Técnica, sendo Diretor, o Dr. Marcos Augusto Enrietti. A mesma situação ocorreu com o volume XII (1966) sendo Diretor o Dr. Rolando Sallin Zappa Mansur, e com o volume XIII (1967/1970), sendo Diretor o Dr. Alsedo Leprevost. Do volume XIV (1971), agora sendo Diretor o Dr. Dinor Olegário Voss, consta o primeiro Conselho Editorial de Arquivos e uma Comissão Especial de Redação. O volume XV (1972) aparece com sua nova capa em verde, branco e preto, mantida até o volume 39 (1996). por 24 anos, portanto, com absoluta regularidade de publicação. Nesse período, foram Editores de Arquivos de Biologia e Tecnologia, o Prof. Luiz Alberto Silva Veiga (vol. XV (2), 1972 ao vol. 22 (1), 1979), o Prof. Annibal de Paiva Campello (vol. 23 (1), 1980 ao vol. 28 (3), 1985) quando Diretor o Dr. Dinor Olegário Voss. a partir do vol. 28(4), sendo Diretor do IBPT o Dr. Edmundo Reichmann, Diretor Administrativo, Luis Carlos Blanc e Diretor Técnico, o Dr. Aurelino Menarin Jr. o Prof. Fridolin Schlögel passa a ser o Editor, prestando importante contribuição à regularidade e ao alto nível de Arquivos de Biologia e Tecnologia, com o qual contribuiu até o vol 40(3), quando Diretor do Instituto de Tecnologia do Paraná o Dr. Alexandre Fontana Beltrão e Diretor Técnico o Dr. Júlio C, Felix. A partir do vol, 40(3) 1997, agora já no vol. 44(2) 2001, Arquivos de Biologia e Tecnologia é internacionalizado passando a Brazilian Archives of Biology and Technology, sendo Editor o Prof. Carlos Ricardo Soccol.

É fato muito interessante que merece ser considerado, quando se procede a uma análise global da atividade desenvolvida por Arquivos de Biologia e Tecnologia ao longo de sua trajetória de revista científica, é o reflexo que teve em suas publicações o surgimento e o desenvolvimento de novos campos de pesquisa.

Em relação a esse detalhe, nota-se uma interessante curiosidade quando da organização do Conselho Editorial que figura no volume 15, de 1972, editado pelo Prof. Luiz Alberto Silva Veiga, ocasião em que as atividades de pesquisa na área da Bioquímica tinham atingido nível nacional e internacional, além da oferta anual que a Divisão de Bioquímica fazia do notável Curso de Fisiologia de Microorganismos, tudo isso somado a uma produção científica traduzida em ponderável número de trabalhos publicados e de comunicações 
a reuniões e simpósios. Justifica-se, assim, a razão pela qual dos vinte e um membros do Conselho Editorial que figura naquele volume, onze eram oriundos da área da Bioquímica do IBPT e de outras entidades de pesquisa do País: Annibal de Paiva Campello, Déa Amaral, José H. Duarte, Dinor O. Voss, na ocasião também Diretor do IBPT, Glací Zancan, Jahyr Campello, João Baptista Corrêa, Luiz R. Travassos, Marco Aurélio Lacombe Feijó, Marcos Marés-Guias e Ricardo Brentani.

Essa intensa atividade na área da pesquisa bioquímica teve outro importante reflexo no alto significado que atingiu Arquivos de Biologia e Tecnologia, no contexto geral da pesquisa científica no Brasil, tendo publicado, durante 1980 e 1989, um dos números de cada um dos volumes 23 a 32 , as comunicações científicas e os resumos de simpósios apresentados às respectivas Reuniões da Sociedade Brasileira de Bioquímica, bem como de reuniões regionais - II Reunião Regional da $\mathrm{SBBq}$ do Recife (1982) e da Reunião Regional do Nordeste da SBBq (1987), totalizando 3799 resumos publicados.

No que concerne à Biologia Marinha, implantada, entre nós, com a criação do Centro de Biologia Marinha, hoje Centro de Estudos do Mar, da Universidade Federal do Paraná, houve importante reflexo em relação ao número de contribuições oriundas de pesquisas dessa área bem como da área referente à biologia de águas continentais, com ponderável número de trabalhos publicados. O primeiro deles foi o da Dra. Monica Montu, Reproducción y ciclos de vida en quatro especies de eufausídicos patagônicos (Crustacea, Eucaridae), Arq. Biol. Tecnol. 25(2):247-259, 1982.
Vale mencionar, também que Arquivos de Biologia e Tecnologia começou a ter caráter internacional, com o recebimento do primeiro trabalho do exterior submetido para publicação, cujos autores, os Drs. M.T Gómez-Puyou e A. Gómez-Puyou, pertenciam ao Centro de Investigações em Fisiologia Celular da Universidade Nacional Autônoma do México : Gómez-Puyou, M.T. e Gómez-Puyou, A., On the structure, function and regulation of Proton ATPases, Arq. Biol. Tecnol., 24(2):297-221, 1981. Importante iniciativa de Arquivos de Biologia e Tecnologia foi a de publicar um número especial do vol. XIV (1985) ao "Simpósio Aberto em Homenagem a Louis Pasteur" promovido pelo Instituto de Biologia e Pesquisas Tecnológicas para comemoração dos "Cem anos após a primeira vacinação antirrábica humana". Ocorreu essa vacinação a 6.7. 1885, no garoto Joseph Meister.

De modo muito significativo, aquela publicação especial de Arquivos de Biologia e Tecnologia relembra que certa feita, Claude Bernard disse a Pasteur: "Os inimigos são os parasitas científicos, impotentes para criar alguma coisa por si mesmos e que, ordinariamente, se agarram às descobertas dos outros para atacá-los e procurarem, deste modo, a ocasião de fazerem com que falem deles".

\section{A PRODUÇÃO CIENTÍFICA}

Uma análise da produção científica do Instituto de Biologia e Pesquisas Tecnológicas, acrescida de contribuições oriundas de outras entidades de pesquisa do País e do exterior atingiu a significativa publicação de 1672 originais que relacionados com áreas de pesquisa que abrangem, totalizam 1861 contribuições (Tabela 1 e Gráfico 1). 
Tabela 1 - Levantamento, por áreas, das contribuições científicas publicadas em Arquivos de Biologia e Tecnologia e Brazilian Journal of Biology and Technology

\begin{tabular}{|c|c|c|c|c|c|}
\hline \multirow{2}{*}{\multicolumn{2}{|c|}{ ÁREAS / SUBÁREAS }} & \multicolumn{3}{|c|}{ VOLUMES } & \multirow{2}{*}{$\begin{array}{c}\text { SUB- } \\
\text { TOTAL }\end{array}$} \\
\hline & & 1 ao 25 & 26 ao $40(3)$ & $40(4)$ ao 44 & \\
\hline & Leishmaniose & 5 & - & 1 & 6 \\
\hline & Oncologia & 2 & 1 & 1 & 4 \\
\hline & Endocrinologia & 5 & 2 & - & 7 \\
\hline PATOLOGIA & Histopatologia & 6 & 1 & - & 7 \\
\hline \multirow[t]{3}{*}{ EXPERIMENTAL } & Histoquímica & 6 & - & - & 6 \\
\hline & Histologia de Invertebrados & 2 & - & 2 & 4 \\
\hline & Fisiologia e Fisiopatologia de Vertebrados & - & 29 & 5 & 34 \\
\hline \multicolumn{2}{|c|}{ FISIOLOGIA DE INVERTEBRADOS } & - & 12 & 5 & 17 \\
\hline \multicolumn{2}{|c|}{ BIOQÚIMICA } & 133 & 109 & 57 & 249 \\
\hline \multicolumn{2}{|l|}{ BIOTECNOLOGIA } & 8 & 121 & 28 & 157 \\
\hline \multicolumn{2}{|c|}{ FISIOLOGIA DE MICROORGANISMOS } & 7 & 37 & 12 & 56 \\
\hline \multicolumn{2}{|c|}{ GENÉTICA DE MICROORGANISMOS } & 1 & - & - & 1 \\
\hline \multicolumn{2}{|c|}{ FÍSICO-QUÍMICA } & 5 & 15 & 2 & 22 \\
\hline \multicolumn{2}{|c|}{ GEOLOGIA E PALEONTOLOGIA } & 28 & - & - & 28 \\
\hline \multicolumn{2}{|c|}{ GEOGRAFIA e PESQUISAS GEOGRÁFICAS } & 6 & - & - & 6 \\
\hline \multicolumn{2}{|c|}{ ARQUEOLOGIA } & 1 & - & - & 1 \\
\hline \multicolumn{2}{|c|}{ MINERALOGIA e RECURSOS MINERAIS } & 21 & 7 & - & 28 \\
\hline & Inorgânica & 10 & 18 & 1 & 29 \\
\hline \multirow[t]{4}{*}{ QUÍMICA } & Orgânica & 11 & 7 & 1 & 19 \\
\hline & Bromatológica & 14 & 53 & 7 & 74 \\
\hline & Botânica & 4 & 18 & 14 & 36 \\
\hline & Bioquímica Vegetal & 8 & 70 & 29 & 107 \\
\hline \multirow[t]{3}{*}{ BOTÂNICA } & Fisiologia Vegetal & 17 & 99 & 33 & 149 \\
\hline & Fitogeografia e Reflorestamento & 3 & 17 & 6 & 26 \\
\hline & Fitossociologia & - & 8 & 2 & 10 \\
\hline \multicolumn{2}{|l|}{ FITOPATOLOGIA } & 28 & 6 & 10 & 44 \\
\hline \multicolumn{2}{|l|}{ ENTOMOLOGIA } & 20 & 33 & 7 & 60 \\
\hline PARASITOLOGIA & & 25 & 10 & 3 & 38 \\
\hline MICROBIOLOGIA & IMUNOLOGIA & 13 & 44 & 9 & 63 \\
\hline & Patologia Animal & 6 & 3 & - & 9 \\
\hline & Patologia de Animais Silvestres & 3 & 2 & - & 5 \\
\hline PATOLOGIA & Patologia de Aves & - & 3 & - & 3 \\
\hline ANIMAL & Morcegos Hematófagos & 1 & 2 & - & 3 \\
\hline & Hematologia de Bovinos & 1 & - & - & 1 \\
\hline & Patologia de Peixes & - & 1 & - & 1 \\
\hline MICOLOGIA e MIC & PATOLOGIA & 11 & 15 & 4 & 30 \\
\hline EDAFOLOGIA e QL & IMICA DE SOLOS & 13 & 17 & 7 & 37 \\
\hline & Rami & 1 & - & - & 1 \\
\hline & Nó-de-Pinho & 1 & - & - & 1 \\
\hline MATÉRIAS & Cimento Portland & 1 & 2 & - & 3 \\
\hline PRIMAS & Mandioca & - & 1 & - & 1 \\
\hline & Materiais Odontológicos & - & 1 & - & 1 \\
\hline MELHORAMENTO & PRODUÇÃO VEGETAL & 6 & 11 & 15 & 32 \\
\hline MELHORAMENTO & PRODUÇÃO ANIMAL & 6 & 9 & 1 & 16 \\
\hline ZOOLOGIA & & 1 & 29 & 3 & 33 \\
\hline ANATOMIA DE AN & MAIS DOMÉSTICOS & 4 & 2 & - & 6 \\
\hline GINECOLOGIA e O & STETRÍCIA VETERINÁRIA & 5 & 2 & - & 7 \\
\hline EPIDEMIOLOGIA & & 10 & 9 & 1 & 20 \\
\hline NUTRIÇÃO & & - & 19 & 3 & 22 \\
\hline AGROTÓXICOS (s & a partir do vol. XIV) & 2 & 12 & - & 14 \\
\hline FARMACOLOGIA I & FARMACOCINÉTICA & 2 & 9 & 1 & 12 \\
\hline $\begin{array}{l}\text { BIOLOGIA MARIN } \\
\text { (publicações começa }\end{array}$ & $\begin{array}{l}\text { A E DE ESTUÁRIOS } \\
\text { no vol. 25) }\end{array}$ & 9 & 124 & 24 & 157 \\
\hline $\begin{array}{l}\text { BIOLOGIA E ECOL } \\
\text { e LAGOAS) E DE O }\end{array}$ & $\begin{array}{l}\text { GIA DE ÁGUAS CONTINENTAIS (RIOS, LAGOS } \\
\text { GANISMOS AQUÁTICOS (PEIXES) }\end{array}$ & - & 9 & 24 & 33 \\
\hline BIOLOGIA E FISIOI & OGIA DE PEIXES & - & 20 & 13 & 33 \\
\hline AQÜICULTURA & & - & 1 & - & 1 \\
\hline
\end{tabular}


(Cont. Tabela 1)

\begin{tabular}{lcccc}
\hline BIOLOGIA MOLECULAR DO GENE & - & 12 & 8 & 20 \\
METODOLOGIA QUÍMICA, TECNOLÓGICA E BIOLÓGICA & - & 43 & 7 & 50 \\
TECNOLOGIA E PROCESSOS TECNOLÓGICOS & - & 6 & 1 & 7 \\
TECNOLOGIA DE ALIMENTOS & - & - & 1 & 1 \\
MUSEU (MALACOLOGIA) & - & 1 & - & 1 \\
HIGIENE & - & 1 & - & 1 \\
\hline
\end{tabular}

Gráfico 1 - Freqüência de trabalhos em Arquivos de Biologia e Tecnologia e Brazilian Journal of Biology and Technology, mostrando a soma das áreas que mais contribuíram para o total de publicações.

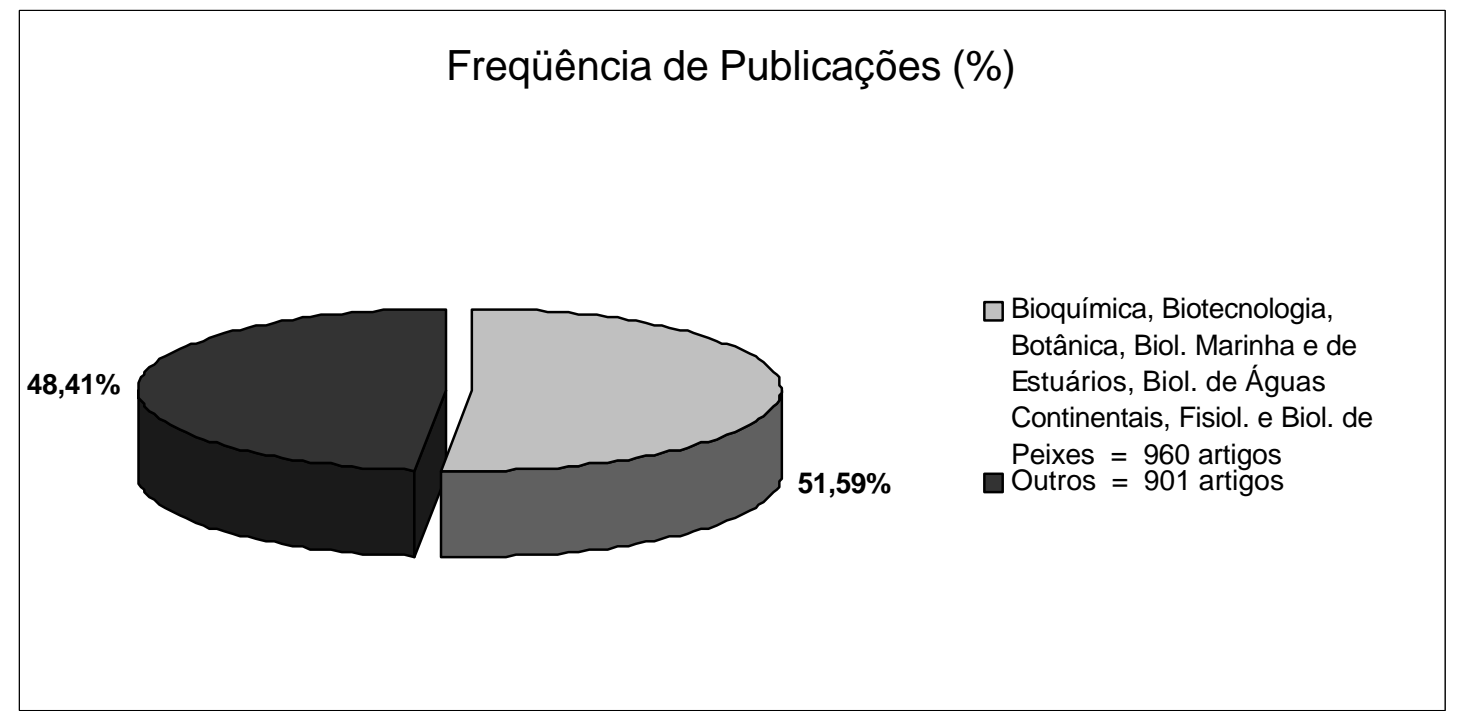

O presente número de Brazilian Journal of Biology and Technology, comemorativo dos 55 anos de publicação de Arquivos de Biologia e Tecnologia, contém as pesquisas de Heitor Medina, sobre leishmanioses e, mais particularmente, sobre a Leishmania enrietti; os trabalhos de Reihnard Maack sobre geologia do Paraná e de Santa Catarina, e os de João José Bigarella sobre a planície litorânea do Estado do Paraná; os estudos sobre os solos dos Campos Gerais no Estado do Paraná de Carlos Bodziak Jr e Reinhard Maack; as contribuições sobre química analítica inorgânica e orgânica de Reinaldo Krauze Spitzner e de Nilton Emílio Bührer; o trabalho de Alsedo Leprevost sobre análise de calcáreos paranaense; as contribuições de Milton Giovanoni e Gastão Victor Langmann Kubiak, cuidando da Fauna parasitológica paranaense e de Lycio Grein de Castro Vellozo, Mario José Nowacki e Milton Miró Vernalha, sobre levantamento fitossanitário do Estado do Paraná; um trabalho de bioquímica enzimológica que marcou o início da "vertente bioquímica" na produção científica do IBPT, de Metry Bacila, sobre galactoquinase em tecidos animais; uma contribuição do Prof. Arthur Otto Schwab, sobre limitações nas medidas físico-químicas; a contribuição de Oscar K. Palmquist sobre brucelose no Estado do Paraná; e enfeixando a publicação um importante trabalho do Dr. Marcos Augusto Enrietti, sobre Leptospirose em murídeos, caninos e suínos no Paraná.

Publicados nos primeiros volumes de Arquivos de Biologia e Tecnologia, os trabalhos selecionados são indicativos das grandes áreas de atuação que caracterizaram as atividades iniciais do Instituto de Biologia e Pesquisas Tecnológicas em suas Divisões de Biologia Animal, de Biologia Vegetal, de Química e Tecnologia, de Geologia e Mineralogia, de Solos, de Patologia Experimental. 


\section{AS LEMBRANÇAS QUE FICARAM}

Um rosário de rememorações do IBPT certamente povoa o repositório sentimental de cada um de nós que tivemos a ventura de viver aquele exuberante momento da vida cientifica de nossa comunidade universitária.

Sem dúvida, a figura exponencial do Dr. Marcos Augusto Enrietti, o nosso estimado Dr. Marcos, sobreleva a tudo e a todos pelo significado de sua atuação e da influência que teve no contexto do movimento científico que tantos frutos gerou entre nós.

Importante testemunho do apreço que os pesquisadores do IBPT tinham para com a estimada figura de Marcos Augusto Enrietti é a sua perpetuação dentro da sistemática zoológica, duradoura e significativa homenagem científica que marcará para sempre a sua memória, como atestam os seguintes trabalhos:

1. MUNIZ, J.; MEDINA, H. Leishmaniose tegumentar do cobaio. Leishmania enrietti. Arq. Biol. Tecn. 3, 13-30, 1948.

2. FERNANDES, B.F.; MOLFI, A Corynosoma enrietti, N. N. Sp., parasita de patos e marrecos domésticos (Paleachantocephala; Polymorphida). Arq. Biol. Tecn. 8, 3-6, 1953.

3. VERNALHA, M.M. Descrição de uma espécie nova do gênero Dichotomius, Hope 1838 (Col. Scarabidae). Arq. Biol. Tecn. 7, 39-42, 1952. (Dedicada ao Dr. Marcos Augusto Enrietti.

Duas homenagens ao Dr. Marcos, que muito o sensibilizaram e aos seus amigos, aconteceram quando o saudoso fundador do IBPT encontravase enfermo. Uma delas, foi a da inauguração do Centro de Diagnóstico Marcos Enrietti. Doente e impossiblitado de locomover-se para tão significativa homenagem, em uma de minhas visitas a Curitiba - na época era Professor Catedrático da Universidade de São Paulo recebi informação de que o Dr. Marcos deseja conversar comigo. Indo à sua residência, fiquei surpreso quando me solicitou que o representasse naquela significativa homenagem. É fácil imaginar a emoção de que fui tomado, quando fiz uma pequena oração, em lugar do Mestre, recebendo aquela que seria, ao meu ver, a mais significativa homenagem que receberia ele, perpetuando o seu nome em instituição daquele porte e significado. Quando voltei a conversar com o Dr. Marcos, entregando-lhe cópia da oração que fizera em seu lugar, a sua emoção foi muito grande.

Outra homenagem que muito o sensibilizou, recebeu ele de amigos e conhecidos seus, que inauguraram um Posto de Gasolina da cidade com o seu nome. Dr. Marcos esteve presente a ato tão singelo mas certamente tão significativo, que revelava a admiração que se tinha por sua ilustre figura humana. Não desejo, neste repositório de lembranças de uma época tão importante para a pesquisa científica entre nós, relembrar as vicissitudes pelas quais passou o Dr. Marcos, em razão dos problemas políticos que teve de enfrentar e nem quero comentar sobre suas origens e da maneira como foram superados. Creio que a verdade histórica é o juiz a quem cabe decidir sobre assuntos dessa natureza, assuntos esses que jamais diminuirão o excelso significado de uma vida tão plena de grandezas como foi a de Marcos Augusto Enrietti.

A pesquisa científica e, certamente o IBPT, cuja história estamos rememorando aqui, teve também os seus mártires. E no caso do IBPT, sempre vem à nossa memória o sacrifício de uma vida em contraste com os benefícios que prestou à humanidade, na figura de nosso sempre lembrado Prof. Dr. Oscar Krebs Palmquist. Um microbiologista de grandes méritos, Palmquist realizou um estudo de fundamental importância para a população paranaense, sobre o grave problema da brucelose que afetava o rebanho leiteiro do Paraná. Tragicamente, porém, o nosso saudoso colega foi contaminado pela brucelose, tendo perdido a vida em consequiência dessa grave infecção que sofrera. Um lado trágico desse infausto evento foi que ele fizera o seu próprio diagnóstico, acompanhando a evolução dos dados laboratoriais relativos ao seu próprio caso.

$\mathrm{E}$, para concluir este tópico de rememorações, uma história que se perpetuou no IBPT tornando-se quase lendária.

Nos próprios do Instituto e em frente à séde da Escola Superior de Agricultura e Veterinária, no Juvevê, há um campo de futebol, ainda muito utilizado para a prática desse esporte por alunos, professores e funcionários. Certamente, os pesquisadores e funcionários administrativos do IBPT jogavam suas "peladas" em momentos de lazer. Para tanto, organizaram dois times de futebol: um, do prédio 
central e outro, dos demais prédios. O Prof. Heitor Medina, que havia sido campeão sulamericano de lançamento de dardo e participante de duas olimpíadas mundiais, era o centroavante do time dos centrais. Em uma das peladas que ficou gravada na memória de todos, o Dr. Marcos jogava como goleiro do time dos demais prédios que estava desfalcado na ocasião. $\mathrm{Na}$ sequiência da partida, o Dr. Medina dá um "dribling" em um adversário e outro no Dr. Marcos e marca um gol de placa.

A reação do Dr. Marcos para o Dr. Medina:

- Vou te suspender por 15 dias por desacato ao Diretor!

A resposta do Dr. Medina foi imediata:

Pois eu vou te transformar em protozoário!

Contam os desafetos que foi assim que nasceu a Leishmania enrietti ... que percorreu o mundo científico, honrando a ciência brasileira, tal a sua importância para a pesquisa biológica e médica. 